(Aus dem physiologischen Institut der Universität Innsbruck.)

\title{
Zeitmessende Beobachtungen über die Wahrnehmung des sich entwickelnden positiven Nachbildes eines elektrischen Funkens.
}

Von

\section{IT. ซ. Vintsengan und Lustig.}

Hierzu 1 Holzschnitt.

Die Beobachtungen Aubert's und Förster's haben gezeigt, dass der elektrische Funke im Stande ist, ein Nachbild zu erzengen; ersterer hat auch dieses Nachbild einer genauen Untersuchung unterworfen. Ueber die Zeit aber, welche das sich entwickelnde positive Nachbild braucht, damit jene Deutlichkeit erreicht werde, welche nothwendig ist, um dasselbe mit Sicherheit wahrnehmen zu können, möge nun das einwirkende (primäre) Licht ein elektrischer Funke oder irgend ein anderes Licht sein, liegen, so weit uns bekannt ist, keine Angaben vor.

Wir legten uns daher folgende Frage vor: wie viel Zeit verstreicht von dem Augenblicke, in welchem ein momentanes Licht auf die Netzhant einwirkt bis zu jenem, in welchem das positive Nachbild hinreichend deutlich geworden ist, um zum Bewusstsein zu gelangen?

Wir konnten wohl zur Beantwortung dieser Frage schreiten, nachdem wir uns durch einige vorläufige Beobachtungen überzeugt hatten, dass einer von uns (A. Lustig) von einem elektrischen Funken ein deutliches positives Nachbild erhalten konnte, an welchem auch sämmtliche Beobachtungen angestellt wurden.

Um die gestellte Frage zu lösen, war es nothwendig, die Apparate so zu ordnen, dass die Entstehung des elektrischen Funkens und das Auftreten des positiven Nachbildes genau mar-

1) H. A ubert, Ueber die durch den electrischen Funken erzeugten Nachbilder. - J. Moleschott, Untersuchungen zur Naturlehre des Menschen und der Thiere. Bd. V, p. 279. 
kirt wurden. Wir glauben diesen Zweck durch folgende Anordnung der Apparate erzielt zu haben.

Es wurde eine kleine Vorrichtung hergestellt, die wir, der Einfachbeit wegen, die Scheibenvorrichtung $(S c h)$ nennen, welche gestattete, dass in demselben Augenblicke, in welchem der elektrische Funke aus einer grossen Leydner'schen Flasche entlockt wird, auch die Zeiger des Hipp'schen Chronoscopes in Bewegung gesetzt werden. $\mathrm{Zu}$ diesem $\mathrm{Zwecke}$ haben wir auf eine gemeinsame Axe zwei Holzscheiben in einer gegenseitigen Entfernung von ungefähr $14 \mathrm{~cm}$ befestigt. Jede Scheibe trug einen metallenen Stift, und es wurde mit der grösstmöglichsten Genauigkeit getrachtet, dass die beiden Stifte und die Axe der Scheiben sich in derselben Ebene befinden. Das Gestell dieser Scheibenvorrichtung wurde auf einem Tisch derart befestigt, dass nicht bloss die Stifte, sondern auch ein Segment der Scheiben über den Tischrand hervorragten. Ein Stift, den wir der Kürze halber den Entlader nennen, stand mit einem dicken wohl isolirten Leitungsdrahte in Verbindung; der andere Stift hingegen, den wir als den Unterbrecher bezeichnen wollen, hatte den $Z$ weck, eine kleine Contactvorrichtung zu unterbrechen.

Gegenüber dem Entlader, in einer Entfernung von ungefähr 10-12 mm, befand sich der Knopf des inneren Beleges einer grossen Leydner'schen Flasche $(L)$.

Gegenüber dem Unterbrecher dagegen und ein wenig höher als der Mittelpunkt des Knopies der Flasche wurde der Contactbebel aufgestellt. Dieser war nun so beschaffen, dass in dem Augenblicke, in welchem der Unterbrecher das eine Ende des Hebels berübrte, am anderen ein guter Contact aufgehoben und mittelst einer in geeigneter Weise angebrachten Feder eine Wiederherstellung desselben verhindert wurde.

Der Contacthebel war in einer guten Nebenleitung des elektrischen Hauptkreises in passender Art eingeschaltet, wie es später noch angegeben werden soll.

Der Draht, welcher vom Entlader ausging, wurde in ein kleines, wohl geschwärztes Kästchen eingeleitet und endete in eine kleine Kugel aus Messing. Dieser gegenuiber in einer Entfernung von ungefähr 4 bis $6 \mathrm{~mm}$ befand sich ein zweites Kügelchen, welches ebenfalls mit einem Leitungsdrahte in Verbindung stand, der zum äusseren Belege der Leydner'schen Flasche ging. Letztere 
konnte mit einer Holtz'schen Influenzelektrisirmaschine geladen werden. Wenn nun der Entlader sich gegeniiber dem Knopfe des inneren Beleges befand, dann sprang der Funke zwischen jenen und den Entlader und gleichzeitig auch zwischen die in dem kleinen Kästchen eingeschlossenen Kugeln; nur der Funke, welcher zwischen diesen beiden übersprang, wurde von dem Reagirenden gesehen.

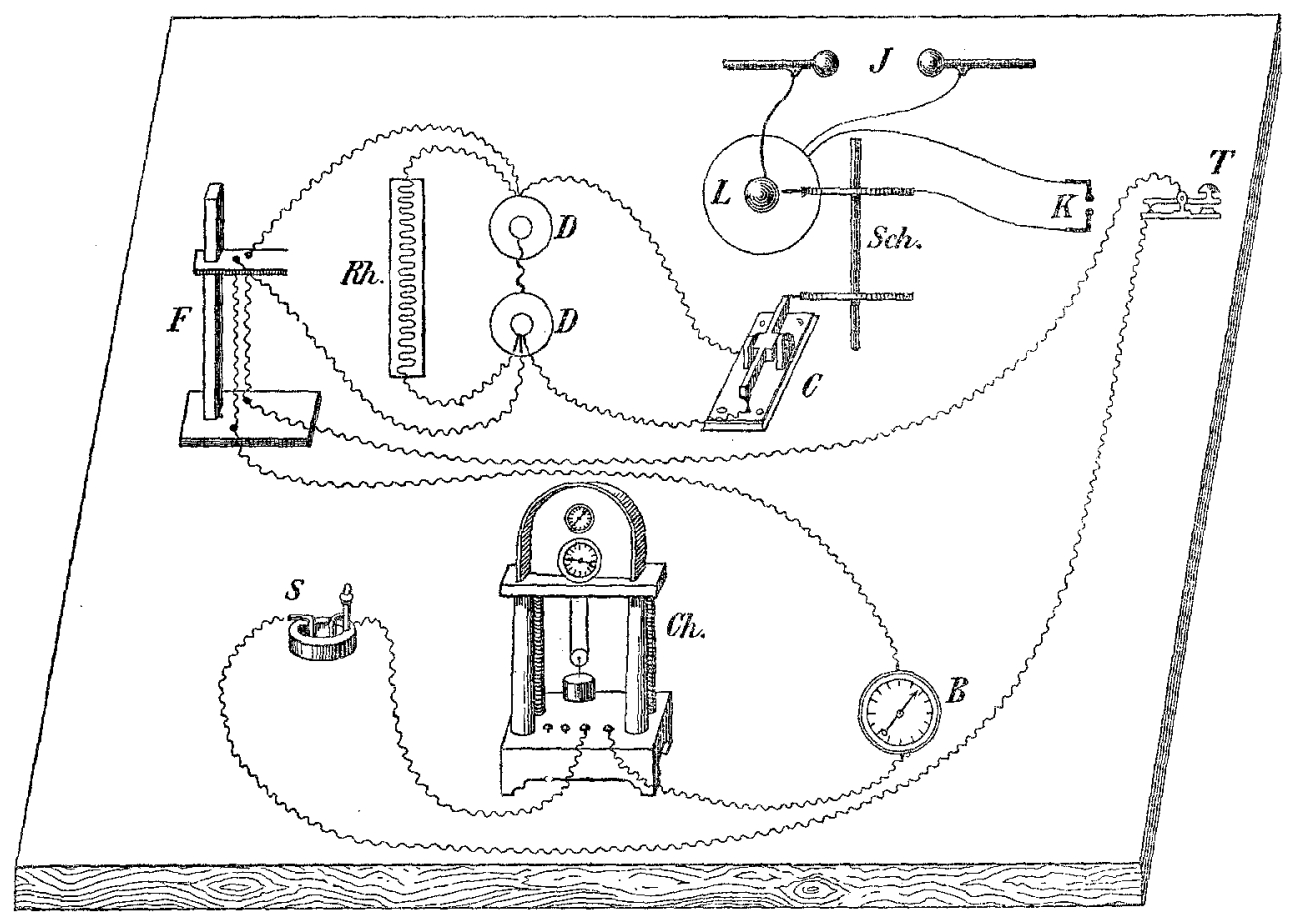

Der zeitmessende Strom wurde von zwei grossen Daniell'schen Elemente $(D)$ geliefert. In der Hauptleitung ${ }^{1}$ ) waren der Reihe nach folgende Apparate eingeschaltet:

1) der Fall-Apparat nach Hipp $(F)$,

2) der Taster für den Reagirenden $(T)$,

3) ein Quecksilberschlüssel $(S)$,

4) das Chronoscop von Hipp $(C h)$ und endlich

5) eine kleine Bussole $(B)$.

1) Vgl. die halb schematische Zeichnung, in welcher die Influenzelectrisirmaschine $(J)$, wie auch das Kästchen $(K)$ und der später zu erwähnende Schirm weggelassen wurden. 
In einer Nebenleitung befand sich das Stöpselrheostat von Siemens $(R h)$ und in einer zweiten der oben erwähnte Contacthebel $(C)$.

Mit dieser Anordnung war es nun ohne wesentliche Aenderung derselben möglich, die Genauigkeit des Chronoscopes zu prïfen und die gewünschten Zeitmessungen vorzunehmen.

Wenn nämlich der Contacthebel $(C)$ und die Nebenleitungen des Fallapparates offen waren, konnte man an der Bussole $(B)$ die Richtung des Stromes und annäherungsweise auch seine Intensität erfahren und letztere mittelst des Rheostates $(R h)$ reguliren und ausserdem mit dem Fallapparate $(F)$ die Genauigkeit des Chronoscopes $(C h)$ prüfen.

Waren die Nebenleitungen des Fallapparates (wie es in der halbschematischen Zeichnung ersichtlich ist) offen und wurde der Contacthebel geschlossen, so war die Anordnung für eine Beobachtung hergestellt.

Nachdem die Leidner'sche Flasche mittelst der Influenzelektrisirmaschine geladen, und das Uhrwerk des Chronoscopes in Bewegung gesetzt worden war, so genügte nun eine rasche Drehung des Scheibenapparates mit der Hand, um den Entlader vor der Kugel der Flasche vorbei zu bewegen. In demselben Momente, in welchem der Funke übersprang, berïhrte der Unterbrecher den Contacthebel, der zeitmessende Strom ergoss sich durch die Hauptleitung und die Zeiger des Uhrwerkes setzten sich in Bewegung, welche selbstverständlich aufhörte, sobald der Reagirende den Taster niederdriickte.

In der bis jetzt beschriebenen Anordnung: können folgende Fehler vorkommen:

1) Das Chronoscop kann die gewöhnlichen wohl bekannten Fehler verursachen.

Wir haben nun das Chronoscop mittelst des Fallapparates vor Beginn jeder Versuchsreihe geprift und die Beobachtungen erst dann begonnen, wenn die ermittelte Zeit nur um 0,001-0,003 S. von der berechneten abwich; eine solche Prüfung wiederholten wir nach Vollendung jeder Versuchsreihe, niemals fanden wir eine irgendwie nennenswerthe Aenderung.

2) Der Funke konnte auf den Entlader überspringen noch bevor der Unterbrecher die Contactvorrichtung beribrte.

Wenn ein solcher Fehler vorkam, so überstieg derselbe gewiss 
nicht wenige Tausendstel einer Sekunde, wie nachfolgende Erwägungen darthun werden.

Beim sehr langsamen Drehen der Scheibenvorrichtang überzeugten wir uns, dass der Funke bald übersprang, wenn der Unterbrecher die Contactvorrichtung berïhrte, bald wenn jener diese noch nicht erreicht hatte; diese Entfernung betrug aber niemals mehr als $4-8 \mathrm{~mm}$. Dieser Fehler hängt, wenn die Entfernung der einzelnen Theile eine unveränderliche ist, von der Grösse der Ladung der Flasche ab, welche niemals vollkommen gleich sein konnte. Da aber in den meisten Fällen die Ladung der Flasche eine solche war, dass der Funke ïbersprang noch bevor der Entlader sich genau in der Verlängerung des horizontalen Durchmessers des Knopfes der Flasche befand, so haben wir, wie oben angegeben wurde, den Contacthebel etwas höher gestellt als den Mittelpunkt der Kugel, und ausserdem getrachtet, die Scheibenvorrichtung so schnell als möglich zu drehen, so dass auch in jenen Fällen, in welehen der Funke etwas früher übersprang als der Unterbrecher den Contacthebel berührte, die Zeit zwischen Ueberspringen des Funkens und Oeffnung des Contacthebels ungemein kurz ausfallen musste.

Es ist weiter denkbar, dass der Funke erst dann ïbersprang, wenn der Contacthebel schon offen war. Wir glauben aber, dass dieser Fehler niemals vorkam, da die Flaschenladung immer etwas grösser war, als es die Ueberwindung der kleinsten Entfernung des Entladers von der Kugel der Flasche erforderte. Sollte aber ein solcher Fehler vorgekommen sein, dann hätte, aus leicht verständlichen Gründen, der Funke ganz ausbleiben müssen, und wenn er auch ibersprang, dann betrug der Fehler gewiss nicht mehr als wenige Tausendstel einer Sekunde.

Um genaue Beobachtungen iber Nachbilder vorzunehmen, ist es nothwendig einen vollkommen finsteren Raum herzustellen, in welchem sich der Reagirende aufhalten muss; in Ermangelung einer Dunkelkammer mussten wir uns in einer anderen Weise behelfen.

Das kleine oben beschriebene hölzerne Kästchen wurde an einen grossen metallenen Schirm befestigt; genan gegenüber den beiden Kügelchen, zwischen welchen der elcktrische Funke überspringen musste, machten wir in dem Schirme eine kleine runde Oeffnung, deren Durchmesser $15 \mathrm{~mm}$ betrug. Diese Oeffnung wurde 
mit einer vollkommen durehsichtigen Glasplatte geschlossen. An den drei freien Rändern des auf einem Tisch befestigsten Schirmes wurde ein undurchsichtiges Tuch befestigt, welches hinreichend gross war, um nicht bloss den Kopf, sondern auch den Oberkörper des vor dem Schirme sitzenden Reagirenden zu bedecken. Das Tuch reichte wohl nicht bis an den Boden, weil sonst seine Schwere lästig und die Wärme unter demselben dem Reagirenden beschwerlich geworden wäre, da jede Versuchsreihe wenigstens eine halbe Stunde dauerte. Aus dem zuletzt angeführten Grunde wurde auch das Tuch zwischen je zwei Beobachtungen etwas gelüftet.

Die Fensterläden wurden so weit geschlossen, dass nur auf das Chronoscop etwas Licht auffiel. Im ganzen Zimmer herrschte somit ein so schwaches licht, welches eben hinreichend war, um die Apparate zu sehen.

Kurze Zeit vor Beginn jeder Beobachtung nahm der Reagirende das Tuch fester um den Leib und stellte seinen Kopf ungefähr $50-60 \mathrm{~cm}$ weit von dem metallenen Schirme. Erst wenn derselbe mittheilte, dass er nicht den geringsten Lichtschimmer sehe, so dass in seinen Augen gar keine störenden Bilder mehr vorhanden waren und das Gesichtsfeld ziemlich gleichmässig dunkel war, wurde eine Beobachtung vorgenommen.

Es sei noch bemerkt, dass die Oeffnung im Schirme in gleicher Höhe mit den Augen sich befand, und dass das Holzkästchen eine abnehmbare Wand besass, um die Lage der zwei Kügelehen richtig stellen zu können.

Auf dem Tische in dem dunkeln Raume wurde der Taster zur Unterbrechung des Hauptstromes angebracht.

Bei den Vorversuchen, die wir vornahmen, befand sich im grossen Schirme in derselben Höhe der runden Oeffnung eine nadelstichgrosse Oeffnung, damit der Reagirende seinen Augen die nöthige Richtung geben konnte. Es stellte sich aber bald heraus, dass das Licht, welches durch dieses Loch eindrang, fuir den absolut finsteren Raum noch immer zu stark war und das Sehen des Nachbildes störte, so dass wir bei den definitiven Beobachtungen dieses Loch verstopfen mussten. Es zeigte sich hierbei, dass auch ohne dasselbe der Reagirende den Augen die adäquate Richtung und Accommodation geben konnte, um die von dem elektrischen Funken beleuchtete Oeffnung im directen Sehen zu 
beobachten, so dass nur der centrale Theil der Netzhaut dem directen Einflusse der Erregung ausgesetzt war. In der That gab der Reagirende nur in den seltensten Fällen an, dass das Nachbild auf einem Seitentheil der Netzhaut entstanden war. In solchen Fällen zeigte sich auch, dass die Zeit für die Signalisirung des Nachbildes ziemlich lang ausfiel.

Es sei schliesslich noch bemerkt, dass der Reagirende angab, er habe den Funken nicht als solchen geseben, sondern bloss die beleuchtete, kreisförmige Oeffnung, so dass auch das Nachbild nicht eine Linie, sondern eine kreisförmige, beleuchtete Scheibe vorstellte.

Nach dieser Schilderung der Apparate, der Versuchsanordnung and der möglichen Fehler wollen wir den Gang einer Beobachtung schildern.

Der Experimentator schloss zuerst den Contacthebel, dann wurde die Scheibenvorrichtung so gestellt, dass die beiden Stifte ungefähr um 100-110 von dem Contacthebel und von dem Knopf der Leidner'schen Flasche entfernt waren. Sobald der Reagirende meldete, dass er für die Beobachtung vollständig vorbereitet sei (siehe oben p. 499), setzte der Experimentator das Uhrwerk des Chronoscopes und der Diener die Scheibe der Influenzelektrisirmaschine in Bewegung. Nachdem die Handhabe 6--8 Drehungen vollendet hatte, zeigte es dieser mit einer stummen Kopfbewegung an, worauf der Experimentator die schon vorher gefasste Scheibenvorrichtung mit der grösstmöglichsten Geschwindigkeit um $180^{\circ}$ bis $200^{\circ}$ drehte. Der Funke übersprang, die Nebenleitung des Contacthebels wurde entfernt und die Zeiger des Chronoscopes setzten sich in Bewegung. Sobald nun der Reagirende das positive Nachbild in hinreichender Deutlichkeit wahrnahm, drückte er mit der grössten Raschheit den Taster nieder, der Hanptstrom hörte auf und die Zeiger standen still.

Der Taster wurde immer mit dem Daumen der rechten Hand niedergedrückt.

Nun bestand die weitere Aufgabe des Experimentators darin, den Stand der beiden Zeiger abzulesen und die Apparate wieder für eine neue Untersuchung in Ordnung zu bringen.

Durch diese ganze Zeit herrschte im Zimmer die grösste Ruhe; es wurde auch unterlassen der Beobachtung ein Signal vorausgehen za lassen, da der richtige Ton der sehwingenden Feder 
des Chronoscopes ohnehin dem Reagirenden anzeigte, dass nach wenigen Sekunden das Ueberspringen des Funkens erfolgen wïrde.

Während der Reagirende ausruhte und wartete, bis das Nachbild vollständig verschwand, dictirte er einige Angaben iber die vorhergegangenen Beobachtungen, nämlich ïber die ungefähre Grösse des Funkens, über die Grösse der Blendung, über die Deutlichkeit des Nachbildes und endlich ïber die Raschheit, mit welcher er reagirte.

Da bei den geschilderten Beobachtungen der Reagirende nur ein Signal allein gab, so wollen wir die angewendete Methode als jene mit einmaliger Signalisirung bezeichnen.

Damit die Beobachtungen gut gelingen, ist es nöthig, jede zu grosse Bewegung der Augen oder des Kopfes zu vermeiden, um zu verhindern, dass die Nachbilder bei ihrem Entstehen ihre Lage wechseln oder sogar ganz verschwinden. Es ist weiter nothwendig, dass der Funke mittelgross sei, damit derselbe nicht durch eine zu grosse Intensität eine starke Blendung hervorrufe, welche nicht bloss für den betreffenden Versuch nachtheilig ist, sondern auch für den folgenden, indem die Netzhaut eine wesentlich längere Zeit beansprucht, um zu einer relativ geeigneten Ruhe zu gelangen.

Um eben die Netzhaut nicht anzustrengen, beobachteten wir die Vorsicht, in einer Sitzung nur wenige Beobachtungen vorzunehmen und ausserdem haben wir, eingedenk der nachtheiligen Folgen des zu häufigen Beobachtens von Nachbildern, häufig einige Tage ausgesetzt.

Die beleuchtete Oeffnung erscheint in dem positiven Nachbilde scharf begrenzt und ziemlich intensiv gefärbt, nämlich in der Mitte blau, an der Peripherie weiss.

Es sei schliesslich noch bemerkt, dass der Reagirende den Taster genau in dem Augenblicke zu bewegen trachtete, in welchem das Nachbild anfing hinreichend deutlich zu werden.

Es sollen nun die Ergebnisse unserer Beobachtungen näher besprochen werden.

Bei denselben hat sich der Einfluss der Uebung in einer sehr deutlichen and prägnanten Weise gezeigt. Es kann dies uns auch nicht besonders tiberraschen, weil es sich um Signalisirung des Beginns einer Erscheiuung handelt, die im gewöhnlichen Leben selten beobachtet wird, und weil schon die Beobachtung der Nach- 
bilder an und für sich eine nicht geringe Uebung verlangt. Wir haben nämlich die Erfahrung gemacht, dass die Reactionszeit eines Nachbildes sich mit der Uebung wesentlich verringerte, obwohl der Reagirende schon eine gewisse Uebung im Signalisiren anderer physiologischer Erscheinungen erworben hatte, und obwohl bei ihm die Nachbilder sich ungemein leicht entwickeln.

Wir hatten schon mehrere Beobachtungen in der oben beschriebenen Weise vorgenommen und auch ziemlich constante Werthe erhalten; als wir aus Beobachtungen, welche wir in anderer Weise anstellten und die später mitgetheilt werden sollen, den Verdacht schöpften, dass die ermittelten Werthe zu gross seien. Wir haben desshalb noch eine zweite Gruppe von Beobachtungen vorgenommen, welehe unseren Verdacht zur Gewissheit machten. Wir werden die erste Gruppe der Beobachtungen, welche wir Mitte November 1883 angestellt haben, aus dem eben erwähnten Grunde nur summarisch behandeln.

Die Gesammtzahl der Beobachtungen dieser ersten Gruppe beträgt 29, wovon aber nur 25 brauchbar sind; diese ergaben als Mittelwerth $0,566 \mathrm{Sec}$. Die Schwankung der Mittelwerthe der drei Beobachtungsreihen ist nicht sehr ansehnlich, nämlich bloss $0,612-0,510=0,102$. Der mittlere Fehler aus diesen 25 Beobachtungen, nach der Methode von Wund $t^{1}$ ) berechnet, beträgt bloss 0,093 .

Der so geringe mittlere Fehler und der nicht sehr ansehnliche Unterschied in den gefundenen Mittelwerthen der drei Beobachtungsreihen hatte uns anfangs über die Richtigkeit des Gesammtmittelwerthes beruhigt; nur die Thatsache, dass die Schwankung zwischen den einzelnen Beobachtungen $0,744-0,253=0,491$ betrug, trübte etwas unsere Befriedigung.

Nach Vollendung anderer Beobachtungen haben wir, wie schon gesagt, die in der angegebenen Weise vorgenommenen Versuche noch einmal wiederholt.

Von diesen Beobachtungen, - weil wir dieselben als die richtigen ansehen, - geben wir im Anhange die ausfübrlichen Protokolle (Nr. 1), während in der folgenden kleinen Tabelle die Ergebnisse der 4 Beobachtungsreihen zusammengestellt sind.

Die Zahl der gesammten Beobachtungen beträgt 36 , von denen wir aber 7 ansschliessen mussten. Es sind dies jene, welche in den Versuchsprotokollen mit * bezeichnet sind.

Aus nachstehender Tabelle ersieht man, dass der Mittelwerth

1) W. Wundt, Grundzüge der physiologischen Psychologie. Leipzig 1880, Bd. II, p. 223. 


\begin{tabular}{|c|c|c|c|c|c|c|}
\hline \multirow{2}{*}{ Datum. } & \multirow{2}{*}{$\begin{array}{l}\text { Zahl der } \\
\text { guten } \\
\text { Beobach- } \\
\text { tungen. }\end{array}$} & \multirow{2}{*}{$\begin{array}{l}\text { Mittel- } \\
\text { werth. }\end{array}$} & \multirow{2}{*}{$\begin{array}{c}\text { Mittlerer } \\
\text { Fehler. }\end{array}$} & \multicolumn{3}{|c|}{$\begin{array}{l}\text { Schwankung in den ein- } \\
\text { zelnen Beobachtungen. }\end{array}$} \\
\hline & & & & Max. & Min. & $\begin{array}{l}\text { Unter- } \\
\text { sohied. }\end{array}$ \\
\hline 29. November 1883 & 7 & 0,380 & 0,050 & 0,498 & 0,328 & 0,170 \\
\hline 1. December 1883 & 7 & 0,354 & 0,033 & 0,432 & 0,300 & 0,132 \\
\hline 3. December 1883 & 7 & 0,306 & 0,051 & 0,383 & 0,238 & 0,145 \\
\hline \multirow[t]{2}{*}{ 4. December 1883} & 8 & 0,335 & 0,047 & \multirow[t]{2}{*}{0,440} & \multirow[t]{2}{*}{0,239} & \multirow[t]{2}{*}{0,201} \\
\hline & 29 & 0,344 & 0,042 & & & \\
\hline
\end{tabular}

0,344 S. beträgt, welcher wesentlich kleiner ist als der Mittelwerth aus der ersten Reihe; eine Erscheinung, die wir, wie erwähnt, aus der grösseren Uebung hervorgegangen erklären. Aber auch in den vorliegenden Beobachtungsreihen lässt sich der Einfluss der Uebung noch immer deutlich wahrnehmen, da, wie die vorstehende Tabelle zeigt, die Beobachtungen vom 29. Nov. nicht bloss den höchsten Mittelwerth lieferten, sondern auch das Maximum und das Minimum derselben grösser sind als in den übrigen Versuchsreihen.

Wollte man auch die Beobachtungen vom 29. Nov. eliminiren, dann blieben noch immer 22 Beobachtungen, deren Mittelwerthe keinen grossen Unterschied darbieten, nämlich bloss $0,354-0,306$ $=0,048$. Der aus diesen Beobachtungen resultirende Gesammtmittelwerth 0,332 ist gewiss nicht wesentlich verschieden von den fritheren.

Wir glauben desshalb sagen zu können: dass zwischen der Einwirkung des Lichtes eines elektrischen Funkens und der Signalisirung des eben hinreichend deutlich entwickelten Nachbildes eine Zeit von $0,344 \mathrm{~S}$. verstreicht. Es soll hiermit durchaus nicht behauptet werden, dass bei anderen Individuen diese Zeit nicht vielleicht etwas kürzer sein könnte, da, wie obige Tabelle und die Protokolle zeigen, wir auch hie und da kleinere Werthe, nämlich nur $0,24 \mathrm{~S}$. erhalten haben.

Bevor wir in der Schilderung der anderen Beobachtungen, die wir vornabmen, weiter schreiten, wird es nicht überflüssig sein, die Frage aufzuwerfen, aus welchen Zeittheilchen der ermittelte Werth zusammengesetzt ist? Wir glauben darin folgende Zeittheilchen unterscheiden zu können: 
1) Erregung der Netzhaut durch das momentane Licht,

2) Abklingen der Erregung;

3) Entwicklung des positiven Nachbildes,

4) Fortpflanzung dieser Netzhauterregung bis zum Gehirne,

5) Erkennung, dass das Nachbild hinreichend deutlich ist,

6) Entschluss zu reagiren,

7) Uebertragung auf die motorischen Centra,

8) Fortpflanzung dieser Erregung in den motorischen Nerven,

9) Bewegung des Fingers, um das Signal zu geben ${ }^{1}$ ).

Wenn nun gestattet wäre, die Zeittheilchen 1, 4, 5, 6, 7, 8 und 9 gleich jenen Zeittheilchen anzusehen, welche auch in der Reactionszeit auf eine momentane Lichterregung vorkommen, so wïrde dann, nach Ermittlung dieser Zeit, der Unterschied zwischen der Reactionszeit auf ein Nachbild und jener einer Lichterregung, uns die Summe der zwei anderen Zeittheilchen, nämlich das $\mathrm{Ab}$ klingen der Erregung der Netzhaut mehr das Entstehen des Nachbildes, angeben.

Dass in der Reactionszeit einer momentanen Lichterregung die beiden zuletzt erwähnten Zeittheilehen nicht vorkommen, ist gewiss und wir brauchen darüber auch keine weiteren Worte zu verlieren.

Ebenso klar ist, dass in beiden Vorgängen die Zeittheilchen, die wir oben mit 1, 6, 7, 8 und 9 bezeichnet haben, gleich sein werden, so bald das Licht in beiden Fällen momentan ist und womöglich die gleiche Intensität hat.

Fraglich kann nun sein, ob die Fortpflanzungsgeschwindigkeit der Netzhauterregung die gleiche ist, je nachdem dieselbe durch das intensive Licht eines elektrischen Funkens oder durch das eben sich entwickelte positive Nachbild erregt wurde; und fraglich ist es ebenfalls, ob die Erkennung des eben entstandenen Nachbildes mit derselben Raschheit erfolge wie die stattgefundene Erregung der Netzhaut durch den elektrischen Funken.

Da das Nachbild lichtschwächer als das erregende Licht ist,

1) Es ist selbstverständlich, dass wir in der Aufzählung dieser Zeittheilchen, die Fortpflanzung der Netzhauterregung durch das electrische Licht bis zum Gehirne und die Wahrnehmung desselben nicht anführen dürfen, da dieselben in der von uns gemessenen Zeit nicht enthalten sind; diese Vorgänge spielen sich ab, während die Erregung der Netzhaut abklingt and das Nachbild sich entwickelt. 
so muss auch seine Erkennung etwas langsamer erfolgen als jene des einfachen Lichteindruckes, so dass in dem oben angedeuteten Untersehiede höchst wahrscheinlich auch ein Bruchtheil jenes Zeittheilchens enthalten sein dürfte, welcher zur Erkennung des Nachbildes erforderlich ist. Wir haben aber kein Mittel, um diesen Bruchtheil zu ermitteln.

Es ist weiter wahrscheinlich, dass die Fortpflanzungsgeschwindigkeit in Folge der Erregung durch einen elektrischen Funken etwas grösser sei als jene in Folge des eben sich entwickelnden Nachbildes, weil erstere gewiss stärker als letztere ist. Die Frage lässt sich aber theoretisch nicht entscheiden, da alle Voraussetzungen, die wir machen könnten, nur hypothetischer Natur wären; eine experimentelle Entscheidung in diesem speciellen Falle ist aber ausgeschlossen. Wenn auch ein Unterschied vorhanden sein sollte, so ist derselbe gewiss so versehwindend klein, dass er wohl vernachlässigt werden kann.

Die eben angeführten Erörterungen zeigen uns, dass, wenn man von der Reactionszeit eines positiven Nachbildes jene einer Lichtempfindung abzieht, vorausgesetzt, dass alle übrigen Versuchsbedingungen unverändert bleiben, der Unterschied der Summe folgender Zeittheilchen gleich sein wird:

1) der Zeit des Abklingens der Netzhauterregung;

2) der Zeit bis das Nachbild hinreichend deutlich geworden ist und wahrscheinlich

3) einem Bruchtheile der Zeit, welche zur Erkennung der Deutlichkeit des Nachbildes erforderlich ist.

Um nun die Reactionszeit einer Lichtempfindung zu bestimmen, benützten wir dieselbe Anordnung der Apparate, welche in den früheren Seiten beschrieben wurde, und beobachteten die nämlichen Vorsichten, um einen vollkommen finsteren Raum zu haben; auch der Gang einer jeden Beobachtung blieb sich derselbe, wie für die Bestimmung der Reactionszeit des Nachbildes, nur mit der wohl selbstverständlichen Modification, dass nun der Reagirende den Hauptstrom unterbrach, sobald er den elektrischen Funken wahrnahm.

Die erzielten Resultate sind in nachstehender Tabelle zusammengestellt: 


\begin{tabular}{|c|c|c|c|c|c|c|}
\hline \multirow{2}{*}{ Datum. } & \multirow{2}{*}{$\begin{array}{c}\text { Zahl der } \\
\text { guten } \\
\text { Beobach- } \\
\text { tungen. }\end{array}$} & \multirow{2}{*}{$\begin{array}{l}\text { Mittel- } \\
\text { werth. }\end{array}$} & \multirow{2}{*}{$\begin{array}{l}\text { Mittlerer } \\
\text { Fehler. }\end{array}$} & \multicolumn{3}{|c|}{$\begin{array}{l}\text { Schwankung in den ein- } \\
\text { zelnen Beobachtungen. }\end{array}$} \\
\hline & & & & $\operatorname{Max}$. & Min. & $\begin{array}{l}\text { Unter- } \\
\text { schied. }\end{array}$ \\
\hline 9. Nov. $1883 \mathrm{~N}$. & 9 & 0,151 & 0,020 & 0,185 & 0,121 & 0,064 \\
\hline 10. Nov. $1883 \mathrm{~V}$. & 14 & 0,185 & 0,018 & 0,171 & 0,091 & 0,080 \\
\hline 14. Nov. $1883 \mathrm{~V}$. & 8 & 0,143 & 0,011 & 0,180 & 0,111 & 0,069 \\
\hline 15. Nov, 1883 V. & 8 & 0,123 & 0,016 & 0,153 & 0,101 & 0,052 \\
\hline 16. Nov. $1883 \mathrm{~V}$. & 11 & 0,134 & 0,025 & 0,179 & 0,101 & 0,078 \\
\hline \multirow[t]{2}{*}{ 17. Nov. 1888 V. } & 5 & 0,127 & 0,011 & \multirow[t]{2}{*}{0,140} & \multirow[t]{2}{*}{0,112} & \multirow[t]{2}{*}{0,028} \\
\hline & 55 & 0,136 & 0,019 & & & \\
\hline
\end{tabular}

In vorliegender Tabelle fehlt die erste Versuchsreihe, weil der Reagirende noch nicht die hinreichende Uebung hatte; wir haben ausserdem aus den mitgetheilten Versuebsreiben alle jene Beobachtungen eliminirt, bei welchen entweder der Reagirende selbst angab nicht rasch genug reagirt zu haben, oder weil die erhaltene Reactionszeit im Vergleiche zu jener der übrigen Beobachtungen zu gross ausfiel. Es wurden aber im Ganzen nur 10 Beobachtungen eliminirt. Aus den 55 guten Beobachtungen wurde als Mittelwerth $0,136 \mathrm{~S}$. berechnet mit einem mittleren Fehler von nur 0,019. Die Schwankung in den Mittelwerthen der einzelnen Reihen ist nicht gross, sie beträgt $0,151-0,123=0,028$; dagegen ist jene der einzelnen Beobachtungen ziemlich ansehnlich, nämlich $0,185-0,091=0,094$. Es muss aber ausdrücklich erwähnt werden, dass solche extreme Werthe nur höchst selten vorkommen.

Wenn wir nun die für die Lichtempfindung gefundene Reactionszeit $(0,136 \mathrm{~S}$.) von jener für das positive Nachbild $(0,344 \mathrm{~S}$.) abziehen, so bleiben 0,208 S., welche der Summe der oben S. 505 erwähnten Zeittheilchen entsprechen würde.

Wir haben unsere zeitmessenden Beobachtungen auch in einer anderen Weise vorgenommen.

Bei den in den vorhergehenden Seiten beschriebenen Versuchen über die Reactionszeit eines Nachbildes nahmen wir als Anfangsmoment der zu messenden Zeit das Entstehen des Funkens (Erregung der Netzhaut) und als Endpunkt die Wahrnehmung des eben hinreichend entwickelten Nachbildes. 
Letzter Punkt lässt sich nicht ändern, wohl aber ist es möglich, als Ausgangspunkt das Signalisiren der Lichtempfindung zu benützen, und in diesem Falle wäre die gemessene Zeit zwischen Signalisiren der Lichtempfindung und jener des eben hinreichend entwickelten positiven Nachbildes eingeschlossen. Die Apparate müssen daher so angeordnet werden, dass, wenn der Reagirende die Lichtempfindung signalisirt, die Zeiger des Chronoscopes sich in Bewegung setzen, und deren Arretirung in dem Augenblicke erfolge, in welchem das entwickelte Nachbild signalisirt wird. $\mathrm{Zu}$ diesem Zwecke wurden die Apparate folgendermaassen angeordnet:

Die Contactvorrichtung $(C)$ wurde ausgeschaltet, und dagegen in dieser Nebenleitung ein Taster eingeschaltet, welcher sich in der Nähe des Reagirenden befand; sobald nun dieser Taster niedergedritickt wurde, ergoss sich der Strom in die Hauptleitung und die Zeiger des Chronoscopes setzten sich in Bewegung; der Taster $(T)$ in der Hauptleitung befand sich ebenfalls wie vorher in der Nähe des Reagirenden und beim Niederdrücken desselben, wäbrend der erste durch die ganze Zeit offen gehalten wurde, geschah die Unterbrechung der Hauptleitung, worauf die Zeiger des Chronoscopes nun still standen. Die Scheibenvorrichtnng. diente gegenwärtig nur dazu, um aus der hinreichend geladenen Flasche den Funken zu entlocken. Wir möchten diese Methode als jene der zweimaligen Signalisirung bezeichnen ${ }^{1}$ ). Alle übrigen Versuchsbedingungen waren nun auch bei diesen Beobachtungen genall dieselben wie bei den frïheren.

Bei dieser Anordnung entfiel selbstrerständlich der kleine Fehler, der durch das möglicherweise nicht Zusammenfallen der Entstehung des Funkens mit dem Oeffnen des Contacthebels bei

1) Ausser der im Texte erwähnten Methode benutzten wir noch eine zweite einfachere, bloss mit einem Taster in einer Nebenleitung, so dass, wenn der Reagirende diesen Taster niederdrückte, der Hauptstrom hergestellt wurde and die Zeiger des Chronoscopes sich in Bewegung setzten. In dem Augenblicke, in welchem das Nachbild deutlich genug auftrat, liess der Reagirende den Taster los, die Nebenleitung wurde wieder hergestellt, die Zeiger des Chronoscopes wurden in ihrem Laufe arretirt. Die mit dieser Methode angestellten Beobachtungen haben wir nicht benützt, da die erhaltenen Werthe, aus Gründen, die hier nicht weiter erörtert werden sollen, nicht verlässlich waren. 
den früheren Beobachtungen hätte vorhanden sein können; dagegen sind andere Fehler möglich.

Der Reagirende zeigte nämlich die Lichtempfindung durch Niederdriicken des Tasters in der Nebenleitung mit dem Daumen der linken Hand an, das Erscheinen des Nachbildes dagegen durch Niederdrücken des Tasters in der Hauptleitung mit dem Daumen der rechten Hand. Der Gebrauch der beiden Hände, um zwei Erscheinungen zu signalisiren, kann leicht einen Fehler verursachen, welcher aber mit der Uebung geringer wird, da dieselbe Erscheinung immer mit derselben Hand signalisirt wird.

Ein anderer Fehler kann dadurch entstehen, dass die Aufmerksamkeit des Reagirenden gegenwärtig auf zwei Erscheinungen (Licht, Nachbild) gerichtet sein muss, die hintereinander erfolgen. Auch dieser Fehler kann durch die Uebung kleiner werden. Es muss aber weiter erwähnt werden, dass der letzte Fehler ein verschiedenes Zeichen haben kann, je nachdem die Aufmerksamkeit auf das Erscheinen des Funkens oder anf jenes des Nachbildes mehr gerichtet ist; im ersteren Falle muss die gemessene Zeit etwas länger, im zweiten etwas kürzer ausfallen. Es ist aber denkbar, dass diese kleinen Unterschiede bei Vornahme von mehreren Beobachtungen sich gegenseitig compensiren.

Wir bringen im Anhange Nr. 2 die ausführlichen Protokolle von 30 Beobachtungen, die wir nach der geschilderten Methode ausfïhrten und bemerken, dass wir die allerersten Beobachtungen nicht mittheilen, weil bei denselben die Uebung noch eine zu geringe war.

\begin{tabular}{|c|c|c|c|c|c|c|}
\hline \multirow{2}{*}{ Datum. } & \multirow{2}{*}{$\mid \begin{array}{c}\text { Zahl der } \\
\text { guten } \\
\text { Beobach- } \\
\text { tungen. }\end{array}$} & \multirow{2}{*}{$\begin{array}{l}\text { Mittel- } \\
\text { werth. }\end{array}$} & \multirow{2}{*}{$\begin{array}{l}\text { Mittlerer } \\
\text { Fehler. }\end{array}$} & \multicolumn{3}{|c|}{$\begin{array}{l}\text { Schwankung in den ein- } \\
\text { zelnen Beobachtungen. }\end{array}$} \\
\hline & & & & Max. & Min. & $\begin{array}{l}\text { Unter- } \\
\text { schied. }\end{array}$ \\
\hline 24. November 1883 & 4 & 0,392 & 0,074 & 0,477 & 0,294 & 0,183 \\
\hline 27. November 1883 & 7 & 0,263 & 0,066 & 0,469 & 0,195 & 0,274 \\
\hline 28. November 1883 & 6 & 0,224 & 0,044 & 0,284 & 0,104 & 0,180 \\
\hline \multirow[t]{2}{*}{ 29. November 1883} & 6 & 0,251 & 0,042 & \multirow[t]{2}{*}{0,304} & \multirow[t]{2}{*}{0,182} & \multirow[t]{2}{*}{0,122} \\
\hline & 23 & 0,272 & 0,062 & & & \\
\hline
\end{tabular}


In vorstehender Tabelle stellten wir zur leichteren Uebersicht die 23 Beobachtungen zusammen, die wir zur Ermittlung des Mittelwerthes benitzten; die iibrigen 7 haben wir aus Grinden, die in den Protokollen ersichtlich sind, ausgeschieden.

Aus vorstehender Tabelle ersieht man, dass der Mittelwerth $0,272 \mathrm{~S}$. beträgt. Die Schwankung in den einzelnen Mittelwerthen ist nicht ansehnlich, nämlich $0,392-0,224=0,168$. Der mittlere Fehler ist sehr gering, derselbe beträgt nur 0,062; dagegen ist die Schwankung in den Werthen der einzelnen Beobachtungen sehr gross, nämlich $0,477-0,104=0,373$. Trotz dieser grossen Schwankung glauben wir jedoch, dass der berechnete Gesammtmittelwerth nicht wesentlich von der Richtigkeit abweichen dürfte, da aus den drei letzten Versuchsreihen, welche ziemlich tibereinstimrnende Mittelwerthe ergaben, sich als Gesammtmittelwerth $0,247 \mathrm{~S}$. berechnen lässt, welcher nur um $0,025 \mathrm{~S}$. kleiner ist als der friihere.

In dem eben ermittelten Werthe fehlt gänzlich jene Zeit, welche von der Erregung der Netzhaut bis zu deren Signalisirung verstreicht, und es wäre somit zu vermuthen, dass, wenn man diese beiden Werthe addirt, jene Zeit erhalten werden könnte, welche rom Entstehen des Funkens bis zur Signalisirung des Nachbildes gefunden wurde (Methode der einmaligen Signalisirung). Die eben angedeutete Summe ergiebt:

$$
\begin{aligned}
& \text { Reactionszeit einer Lichtempfindung. . . . . . . }=0,136 \\
& \text { für das Nachbild mit der Methode der zweimaligen } \\
& \text { Signalisirung . . . . . . . . . . . . . . . }=\frac{0,272}{0,408 .}
\end{aligned}
$$

Diese Summe ist um 0,064 S. grösser als der Werth (0,344 S.), der für die Reactionszeit eines Nachbildes (Methode der einmaligen Signalisirung) gefunden wurde.

Es wäre, wie erwähnt, zu erwarten gewesen, dass die Summe, wenn nicht kleiner, doch wenigstens gleich mit der Reactionszeit eines Nachbildes sich bätte herausstellen sollen, weil während des Ablaufens des Processes für die Signalisirung des Lichteindruckes das Abklingen der Netzhauterregung stattfindet, und die Entwickelung des Nachbildes beginnt.

Wir können uns nicht verhehlen, dass es wohl etwas Gewagtes ist, auf einen so kleinen Untersehied von $0,06 \mathrm{~S}$. ein Gewicht $\mathrm{zu}$ legen, nachdem, wie die oben angefuihrten Tabellen zeigen, nicht 
bloss die einzelnen Beobachtungen, sondern auch die Mittelwerthe der einzelnen Beobachtungsreihen Schwankungen darbieten, welche den eben angefiuhrten Unterschied iibersteigen.

Wollte man jedoch eine Erklärung dieses Unterschiedes geben, so glauben wir dieselbe im Folgenden zu finden.

Wenn bloss auf das Nachbild reagirt wird (Methode der einmaligen Signalisirung), ist die Aufmerksamkeit des Reagirenden bloss anf das sich entwickelnde Nachbild gerichtet, so dass dessen Signalisirung erfolgen kann, sobald dasselbe, wenn anch schwach doch hinreichend markirt ist. Bei den Versuchen dagegen mit zweimaliger Signalisirung ist die Aufmerksamkeit des Reagirenden zuerst auf das Erscheinen des Lichtes gerichtet, um darauf dessen Signalisirung so schnell als nur möglich vornehmen zu können, und erst nachher richtet er die Aufmerksamkeit auf das in der Entwicklung begriffene Nachbild.

Man kann wohl vermuthen, dass dieser Uebergang der Aufmerksamkeit von einem objectiven Gegenstande zu einer subjectiven Erscheinung, wenn auch dieselbe nach anssen projicirt wird, eine kleine Zeit in Anspruch nehmen werde. Es ist weiter wahrscheinlich, dass in letzterem Falle das in Entwicklung begriffene Nachbild etwas sehärfer markirt (etwas weiter entwickelt) sein muss, um es mit Sicherheit erkennen zu können.

Aus dem Gesagten muss gefolgert werden, dass wenn der Reagirende bei der Methode der zwelmaligen Signalisirung seine Aufmerksamkeit auf das in der Entwicklung begriffene positive Nachbild richtet, derselbe nicht im Stande ist ein Nachbild zu erkennen, welches nach der Methode der einmaligen Signalisirung höchst wahrscheinlich erkannt worden wäre.

Diese Erklärung würde auch für jenen Fall passen, in welchem die Summe der Reactionszeit eines Lichteindruckes und der Reactionszeit des Nachbildes nach der Methode der doppelten Signalisirung den gleichen Werth gegeben hätte, wie die Reactionszeit des Nachbildes nach der Methode der einfachen Signalisirung. 
Tabelle I.

Nach der Methode der einmaligen Signalisirung.

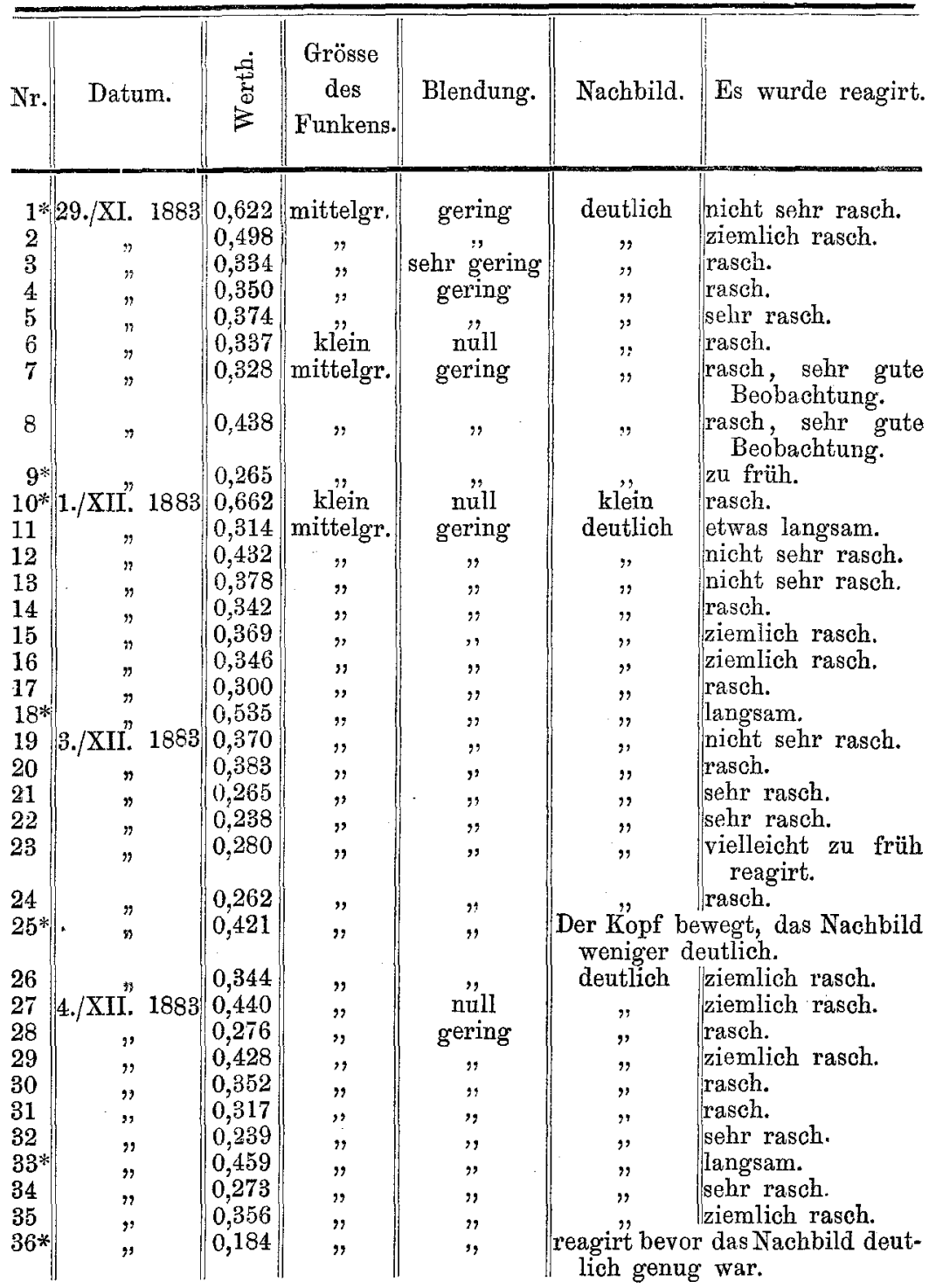


512 M. v. Vintschgau und A. Lustig: Zeitmessende Beobachtungen etc.

Tabelle II.

Nach der Methode der zweimaligen Signalisirung.

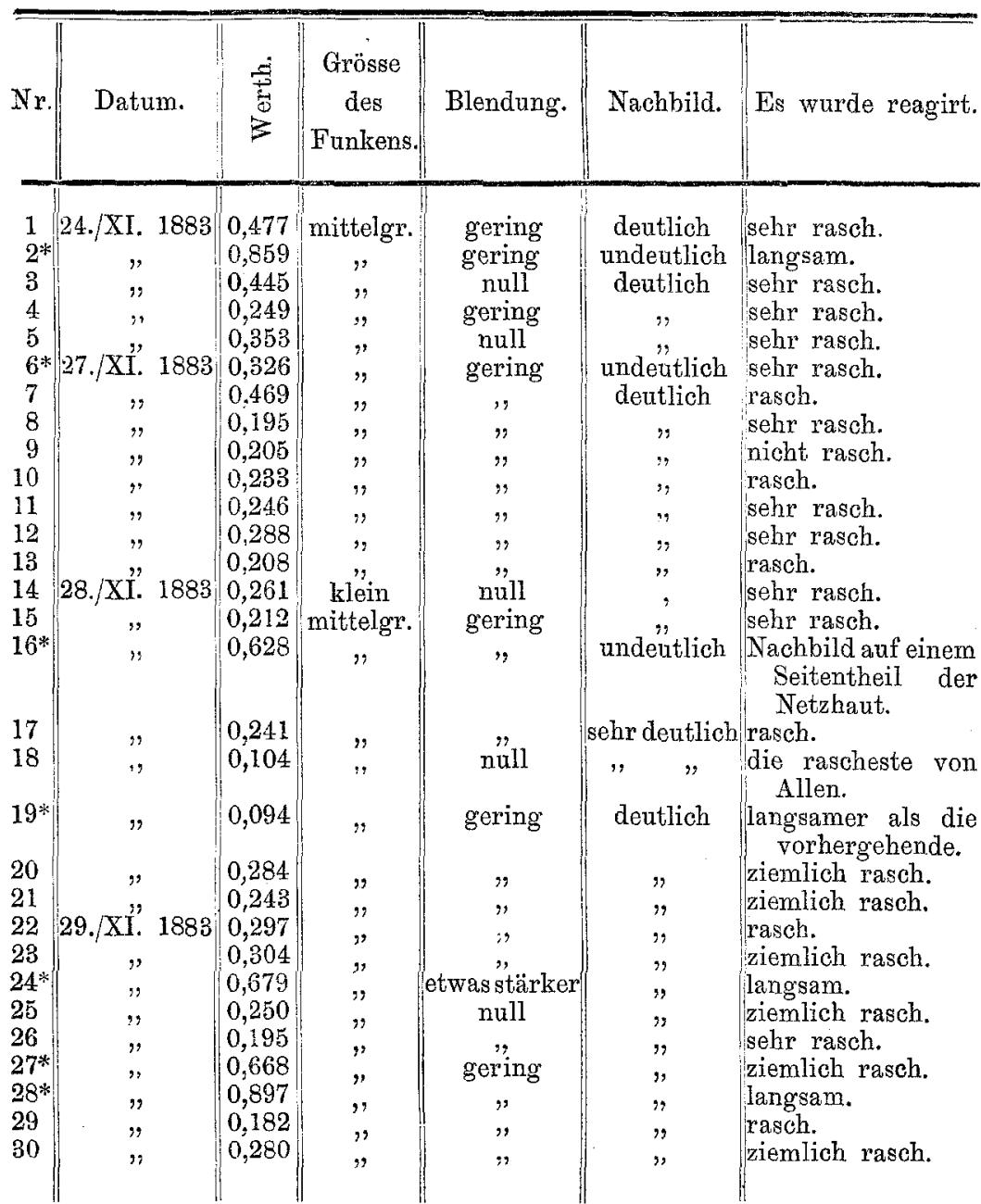

* Alle Beobachtungen mit diesem Zeichen wurden bei der Berechnung des Mittelwerthes vernachlässigt. 